\title{
IMPACT OF THEORETICAL PALLIATIVE CARE TRAINING FOR ONCOLOGY RESIDENTS IN ROMANIA
}

\author{
Daniela Mosoiu¹, Daniel Hinshaw², Timothy J. Moynihan³ , Florian Strasser ${ }^{4}$, \\ Oana Predoiu5, Laura losub 6
}

1 University of Medicine Brasov, Romania; ${ }^{2}$ University of Michigan School of Medicine, Michigan, US; ${ }^{3}$ Mayo Clinic, Rochester, Minnesota, US; ${ }^{4}$ Kantonsspital St. Gallen, Switzerland;

1,5,,6 HOSPICE Casa Sperantei, Brasov, Romania

BACKGROUND: Although palliative care (PC) services are developed in Romania since 1992 at present around $10 \%$ cancer patients receive specialized palliative care. Training oncologist to deliver basic palliative care is a must.

AIM: To develop a theoretical and practical training program for oncology residents based on ESMO/ASCO recommendation. Assess the baseline knowledge of oncology residents regarding palliative care prior to the educational intervention

METHOD: Based on ESMO/ASCO recommendations, PC curriculum for medical oncology was developed and piloted in 5 counties in Romania. Out of 270 oncology residents from Romania, 144 participated in PC theoretical courses (42 hours). Participants received a pre-test before and a post-test after the theoretical training for testing knowledge related to PC. 31 residents finished also the clinical placement and completed a post-test 2 related to knowledge. Means for each topic from pre and post-test were compared. We used Paired Sample T Test.
RESULTS: Main PC domains covered in the multiplechoice examination included pain, non-pain symptom assessment and management, communication , psychosocial and spiritual care, knowledge of/referral to hospice, end-of-life care. From 144 participants to theoretical training, 86 had both pre and post-test completed. Comparing pre and post test scores there was a $\mathbf{7 0} \%$ increase of knowledge related to PC. There were 36 topics -from which 6 topics didn't have paired question in the pre-test, and post-test. Applying Paired Sample T Test, 30 pairs were compared. For 3 pairs (on breaking bad news, fatigue and neuropsychiatric symptoms) the weren't significant acquisitions; for the rest of 27 pairs significant increase in knowledge and reported skills were detected at $p \leq 0.001$ level.

\section{0 residents are trained in Romania for the medical oncology specialty,} in 9 university centers around the country.
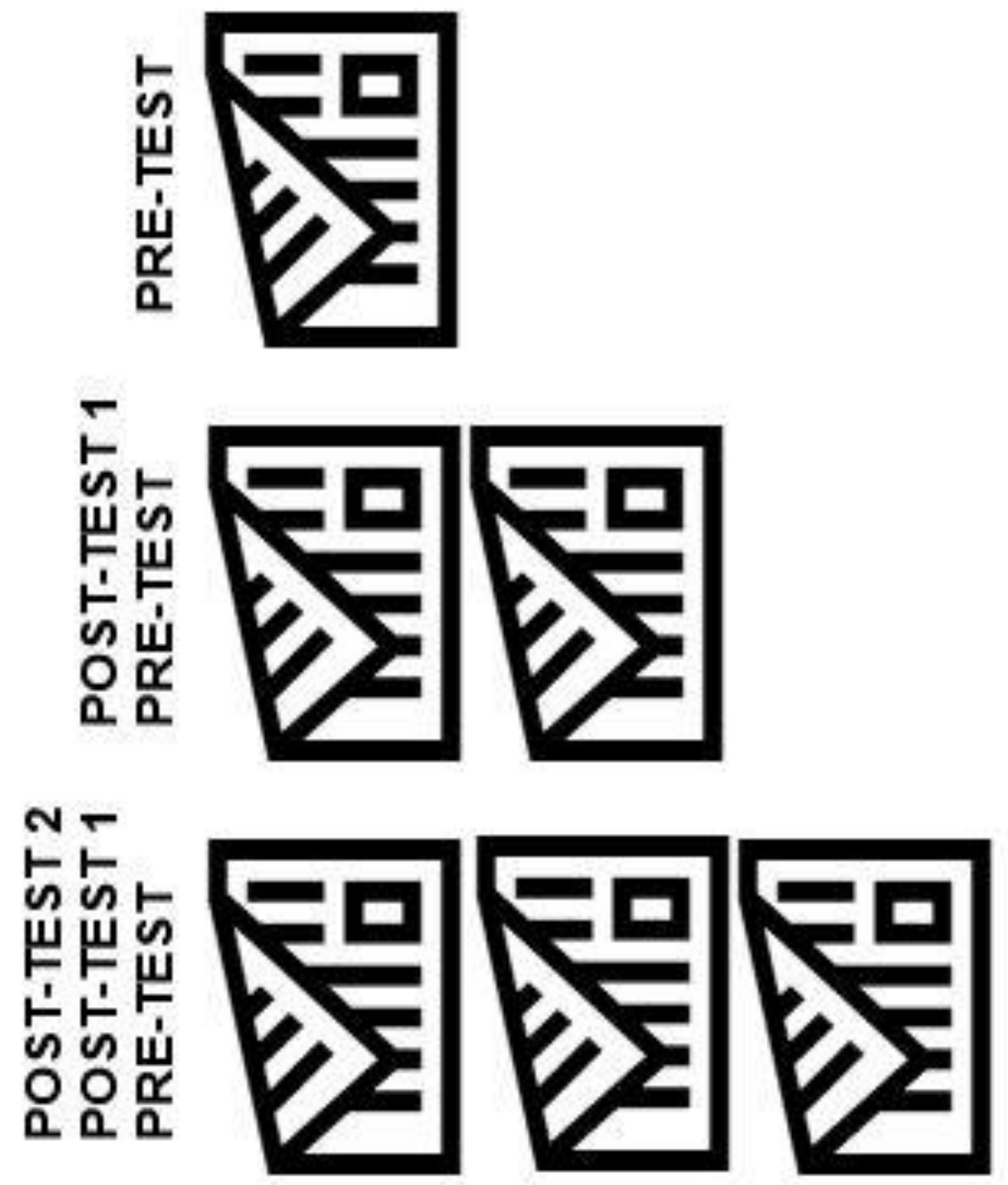
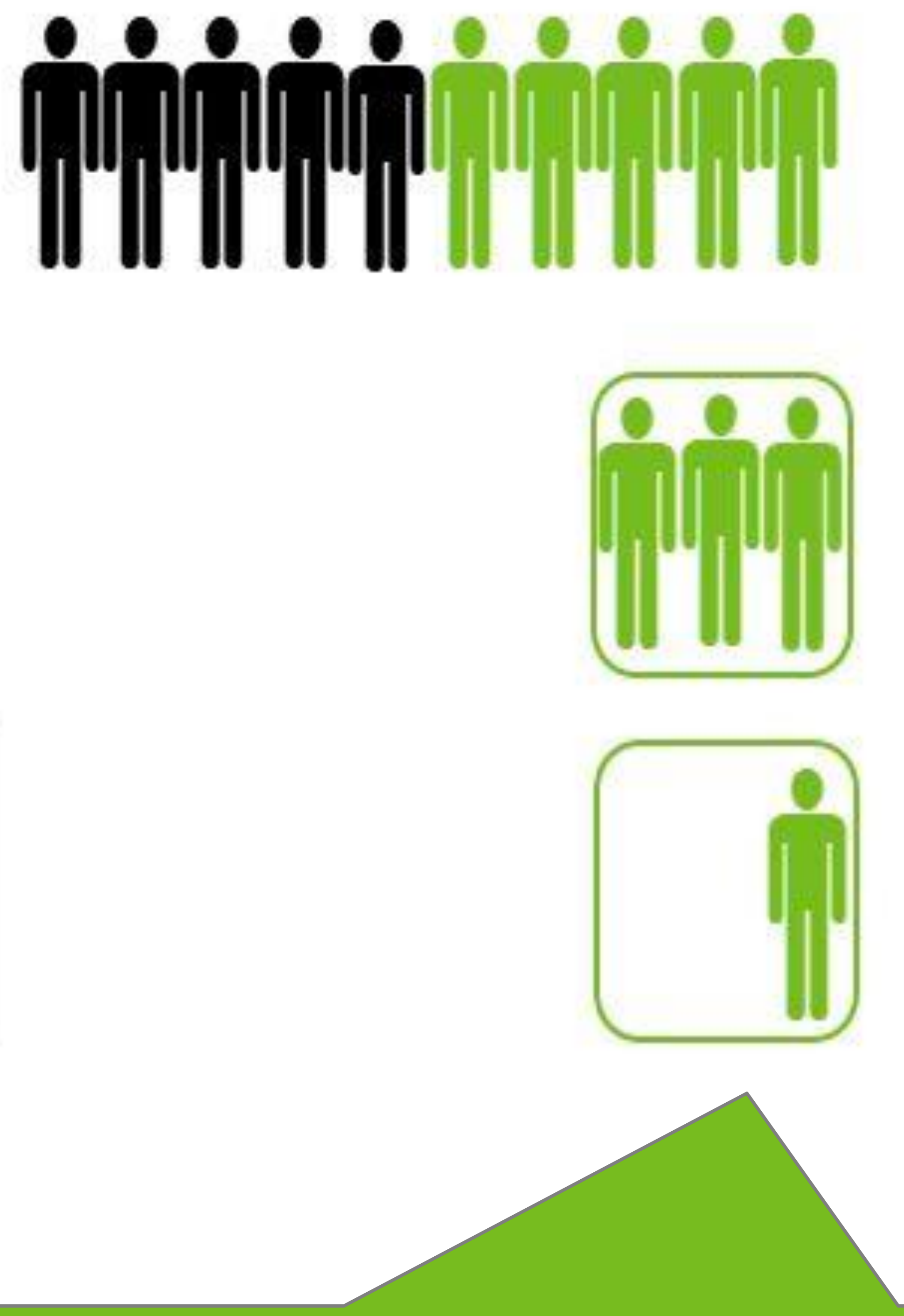

$70 \%$ increase of residents' knowledge related to palliative care topics



144 oncology residents from 5 university centers in the country enrolled in the Palliative Care Theoretical Training PRE-TEST

86 oncology residents graduated the $42 \mathrm{~h}$

Palliative Care Theoretical Training POST-TEST 1

31 oncology residents graduated the 16 weeks

Palliative Care Clinical Placement POST-TEST 2 